\title{
Catalytic hydroxylation enables phenol to efficient assembly of ordered mesoporous carbon under highly acidic conditions
}

Mingjiang Xie, Kai Fang, Yu Shen, Yongzheng Wang, Jiyuan Liang, Luming Peng, Xuefeng Guo*, Weiping Ding

Key Lab of Mesoscopic Chemistry, MOE, School of Chemistry and Chemical Engineering, Nanjing University, Nanjing, P. R. China 210093

* Corresponding authors. Tel.: +86-25-83686393. E-mail addresses: guoxf@ nju.edu.cn (Xuefeng Guo). 


\begin{abstract}
As an important route towards the preparation of ordered mesoporous carbon (OMC), the aqueous assembly method with phenol as precursor still faces a challenge of yielding an OMC under acidic conditions because of the formation of thermoplastic resin in acidic conditions, which leads to structure collapse during carbonization. Herein, we developed an approach combined catalytic hydroxylation of phenol with assembly process to prepare OMC under acidic conditions. In our route, phenol was first partially converted into highly reactive phenols (catechol and hydroquinone) by catalytic hydroxylation, and the resulted mixture of phenols then interacted with template of Pluronic block copolymer F127 under acidic conditions to form a thermosetting mesophase through phase separation, which was carbonized to form OMC. The obtained carbon possesses ordered mesostructure with surface area of 435 $\mathrm{m}^{2} / \mathrm{g}$, pore volume of $0.41 \mathrm{~cm}^{3} / \mathrm{g}$ and achieves high capacitance of $231 \mathrm{~F} / \mathrm{g}$ for supercapacitor compared to that of normal mesoporous carbon (151 F/g) and activated carbon $(\mathrm{AC}, 119 \mathrm{~F} / \mathrm{g})$, which may be attributed to its high oxygen content resulted from catalytic hydroxylation. The developed strategy not only affords a novel approach for the synthesis of OMC but also provides a way to introduce pseudocapacitive oxygen on ordered mesoporous carbon.
\end{abstract}

Keywords: catalytic hydroxylation, phenol, self-assembly, mesoporous carbon, acidic conditions 


\section{Introduction}

Design and synthesis of carbon-based materials with ordered mesoporous structure [1-5] have long been one of the most popular research areas in materials science for theirs unique characters, i.e. ordered porous structure, uniform pore size, large surface area, chemical inertness, good mechanical stability and low cost, which offers promising applications in catalysis [6-9], sorption [10, 11], electrochemical energy storage [12-14] and etc. To construct this type of carbonaceous materials with ordered porous structure, soft template method $[15,16]$ involving a co-assembly between amphiphilic surfactant and the precursor by hydrogen bonding or electrostatic interactions is a prevailing approach in this field.

For soft-templating method, the key to generate ordered mesoporous carbons (OMC) is the precursor, which should be able to assemble with templates to form a threedimensional (3-D) cross-linked network with ordered mesostructure and maintain its ordered mesostructure even after removal of the templates. To date, resorcinol, phloroglucinol and phenol are the commonly used precursors to mesoporous carbon. Resorcinol and phloroglucinol [17-22] can be employed as precursor to assemble OMC under basic or acidic conditions since they possess more reactive site than phenol, favoring formation of 3-D cross-linked network under corresponding conditions. Recently, phenol has attracted extensive attention not only because it is market available, but also because it's appropriate reactivity benefits the assembly with templates [23, 24]. So far, phenol has been the most used precursor for the fabrication of mesoporous carbonaceous materials, while, only when the assembly was operated in a narrow $\mathrm{pH}$ range under aqueous basic conditions, 3-D cross-linked network can be formed and the OMCs $[25,26]$ could be obtained. A novolac resin would be formed when the coassembly of phenol and template was conducted under acidic conditions. Novolac [27, 
$28]$, another type of phenolic resin, is hardly used to prepare OMC [29, 30] since it has a linear chain and thermoplastic properties. It would melt when heated at a high temperature and thus its structure could collapse during carbonization. For this reason, there is few successful report on OMCs synthesized with phenol as precursor under highly acidic conditions. Concerning assembly condition, it is believed that acidic conditions are preferred over basic conditions because the acidic conditions can facilitate and enhance hydrogen and Coulombic interactions between the phenolic resin precursors and the amphiphilic block copolymers [21, 24]. Moreover, the assembly of OMCs under acidic conditions are very important for the functionalization [31] of the mesoporous carbonaceous materials, which may open more application fields for mesoporous carbon-based materials. For example, the aqueous acidic conditions generally favors the introducing of metal ions into mesoporous carbon materials [32].

Hitherto, some endeavors have been devoted to prepare OMC from phenol under acidic conditions by various strategies including adding curing agent (hexamethyltetramine) [29], pre-polymerization [24] under basic conditions and using boron modified phenolic resins as precursor [33]. Alternatively, herein, we developed a simple method designed from catalytic view by introducing active group on phenol to synthesize OMC under acidic conditions. Theoretically, if the phenol was partially converted to highly reactive dihydroxybenzenes (DBs: catechol and hydroquinone) by catalytic oxidation and the mixture of phenol and the obtained DBs was used as precursor, more reactive sites could be introduced to the precursors so that a thermosetting resin with 3D cross-linked network instead of thermoplastic resin could be formed and thus the fabrication of ordered mesoporous carbon could be realized under highly acidic conditions. Based on this strategy, a designed route combined catalytic hydroxylation of phenol and assembly with the template was developed as 
shown in Figure 1. In our designed route, phenol was first partially converted to DBs by a catalytic phenol hydroxylation reaction with hydrogen peroxide as oxidant under mild conditions and then the obtained mixture of phenol, DBs and formaldehyde undergo a co-assembly with the template of F127 under highly acidic conditions to form a thermosetting mesophase by phase separation. Ordered mesoporous carbonaceous materials would be obtained by carbonization of the resulted mesophase at high temperature. To achieve this aim, we employed iron oxide with poor crystallization degree derived from hydrothermal decomposition as catalyst. Iron-based materials have been previously proved to be an active catalyst for phenol catalytic hydroxylation [34] and the poor crystallization degree of the iron oxide benefits its dissolution under highly acidic conditions so that it can be easily removed by phase separation. By the presented way, the assembly towards ordered mesoporous carbonaceous materials under highly acidic conditions with phenol as starting material was realized. The obtained carbon possesses ordered structure with surface area of 435 $\mathrm{m}^{2} / \mathrm{g}$, pore volume of $0.41 \mathrm{~cm}^{3} / \mathrm{g}$ and achieves higher capacitance $(231 \mathrm{~F} / \mathrm{g})$ than normal mesoporous carbon $(151 \mathrm{~F} / \mathrm{g})$, and commercial activated carbon $(119 \mathrm{~F} / \mathrm{g})$ in $1.0 \mathrm{M}$ $\mathrm{H}_{2} \mathrm{SO}_{4}$.

\section{Experimental:}

\subsection{Catalyst preparation}

Iron oxide catalyst was prepared by hydrothermal decomposition of iron nitrate at $353 \mathrm{~K}$ for $12 \mathrm{~h}$. The obtained precipitation was further dried prior to being used as catalyst. The poor crystallization and surface area $\left(37 \mathrm{~m}^{2} / \mathrm{g}\right)$ of the iron oxide catalyst $(\mathrm{FeOx})$ are confirmed by $\mathrm{X}$-ray diffraction pattern and nitrogen sorption isotherms shown in supporting information (SI, Figure S1).

\subsection{Catalytic phenol hydroxylation and assembly of ordered mesoporous carbon}


The catalytic hydroxylation reaction and the following assembly of mesoporous carbon were carried out in a flask. Typically, $0.10 \mathrm{~g}$ iron oxide and $0.94 \mathrm{~g}(0.01 \mathrm{~mol})$ phenol were mixed in $10.0 \mathrm{ml}$ distilled water at temperature of $313 \mathrm{~K}$. The amount of $\mathrm{H}_{2} \mathrm{O}_{2}$ with the molar ratio to phenol ranged from $1 \sim 3.0$ was added into the above reaction system to undergo a catalytic oxidation for 12 hours. Subsequently, $1.2 \mathrm{~g}$ template of Pluronic block copolymer F127 was dissolved in $20.0 \mathrm{ml} 3.0 \mathrm{M} \mathrm{HCl}$ aqueous solution to form a homogeneous solution. Then, the template solution together with $3.0 \mathrm{ml}$ formaldehyde ( $37 \mathrm{wt} . \%$ ) were added to the catalytic reaction solution. The mixture was continuously stirred for 6 hours to undergo co-assembly and phase separation process to obtain a precipitate of the precursors and template. The precipitate was separated and washed with water for several times and dried prior to carbonization. The dried composite was carbonized at 773-973 K (rate of $2 \mathrm{~K} / \mathrm{min}$ ) under Ar flow for 4 hours to remove the template. The obtained mesoporous carbonaceous materials were denoted as MCT-PxHy (MC: mesoporous carbon; T: calcination temperature/K; P: phenol; H: hydrogen peroxide; $\mathrm{x} / \mathrm{y}$ : the molar ratio of phenol to hydrogen peroxide). For comparison, the direct assembly of phenol and template under aqueous highly acidic conditions was also operated without a catalytic hydroxylation process. Typically, $0.01 \mathrm{~mol}$ phenol and $3.0 \mathrm{ml}$ formaldehyde (37 wt. \%) were firstly dissolved in $10.0 \mathrm{ml}$ water. Then, $1.2 \mathrm{~g}$ template of $\mathrm{F} 127$ was dissolved in $20.0 \mathrm{ml} 3.0 \mathrm{M} \mathrm{HCl}$ aqueous solution to form a homogeneous solution. Finally, the precursor solution was added to the template solution to undergo a co-assembly for 120 hours till phase separation occurred. The precipitate was separated and washed with water for several times and dried before carbonization. The dried composite was carbonized under Ar flow at 973 $\mathrm{K}$ (rate of $2 \mathrm{~K} / \mathrm{min}$ ) for 4 hours to remove the template and the obtained carbon was denoted MC973-P1H0. 


\subsection{Characterizations}

X-Ray diffraction (XRD) patterns were recorded on a Philips X'Pert X-ray diffractometer with a $\mathrm{Cu} \mathrm{K} \alpha$ radiation $(40 \mathrm{kV}, 40 \mathrm{~mA})$. Transmission electron micrograph (TEM) images were obtained with a JEOL 2100 microscope operated at $200 \mathrm{kV} . \mathrm{N}_{2}$ sorption isotherms were measured using a Micromeritics ASAP2020 analyzer at $77 \mathrm{~K}$. Before measurements were taken, all samples were degassed at 573 $\mathrm{K}$ for $6 \mathrm{~h}$. The specific surface area is calculated by Brunauer-Emmett-Teller (BET) theory and the pore size distribution is determined by adsorption branch via BarrettJoyner-Halenda (BJH) method. Thermogravimetric and Differential Scanning Calorimetry analysis (TG-DSC) was recorded on NETZSCH STA 449 C under air conditions with the heating rate of $10 \mathrm{~K} / \mathrm{min}$. The catalytic hydroxylation products were analyzed by an Agilent high performance liquid chromatography (HPLC) 1260, using methanol/water (40:60) as mobile phase, C18 column and Ultraviolet detector (277 nm). The molecular formula of reaction products were determined using Agilent 5975 GC/MASS spectrometer. Elemental analysis was carried out with an instrument of vario EL II. FTIR spectra were recorded on a VECTORTM 22 spectrometer. X-ray photoelectron spectra (XPS) were recorded on a Thermo ESCALAB 250 by using $\mathrm{Al}$ Ka radiation $(15 \mathrm{kV}, 150 \mathrm{~W})$. All bindingenergies (BEs) were referenced to the $\mathrm{C} 1 \mathrm{~s}$ peak at $284.6 \mathrm{eV}$.

\subsection{Electrochemical Measurement}

Electrochemical measurements were carried out in a $1.0 \mathrm{M} \mathrm{H}_{2} \mathrm{SO}_{4}$ aqueous electrolyte at room temperature, using a three-electrode cell with an $\mathrm{Ag} / \mathrm{AgCl}$ reference electrode and a platinum coil counter electrode. The testing electrode was prepared by mixing the obtained carbon powder, carbon black and polytetrafluorethylene (PTFE) together at a mass ratio of $85: 10: 5$, and dipping the resulting mixture into steel mesh 
$(1 \mathrm{~cm} \times 2 \mathrm{~cm}$, current collector) before being pressed together at $10.0 \mathrm{M} \mathrm{Pa}$. The electrochemical performances of samples were determined by cyclic voltammetry (CV) and galvanostatic charge/discharge curves. The mass specific capacitance was calculated by the discharge curve according to the formula of $C_{m}=\frac{I \Delta t}{m \Delta V}(\mathrm{~F} / \mathrm{g})$, where I is the current density (A), $\Delta \mathrm{t}$ is the discharge time (s), $\mathrm{m}$ is the weight of active material, $\Delta \mathrm{V}$ is the potential window of discharging $(\mathrm{V})$. As a comparison, commercial obtained activated carbon (AC) with a surface area of $891 \mathrm{~m}^{2} / \mathrm{g}$ and an ordered mesoporous carbon (denoted OMC973-EISA) prepared by EISA method [24] with the surface area of $522 \mathrm{~m}^{2} / \mathrm{g}$ were also investigated for supercapacitor.

\section{Results and discussion}

\section{1 morphology and structure of the final MCTs}

As Figure 2 shown, no diffraction peak emerged in the small angle XRD pattern of MC973-P1H0 and the specific surface area of MC973-P1H0 is $421 \mathrm{~m}^{2} / \mathrm{g}$ contributed mainly by microporosity as listed in Table 1 , indicative of the unfeasibility of direct assembly of phenol and template (F127) to OMC under highly acidic conditions. However, the assembly of OMC could be realized under acidic conditions by introducing catalytic phenol hydroxylation using hydrogen peroxide as oxidant. With the $\mathrm{H}_{2} \mathrm{O}_{2}$ /phenol molar ratio of 2:1 in original catalytic system, the obtained products of MCT-P1H2 (MC: mesoporous carbon; T: calcination temperature/K; P: phenol; H: hydrogen peroxide; 1/2: the molar ratio of phenol to hydrogen peroxide) are taken as example to elucidate the feasibility of the designed route.

To investigate the structural evolution, the obtained product of MCT-P1H2 were carbonized at 773 and 973K, respectively. After template removal under 773 and 973 $\mathrm{K}$, the small angle XRD patterns (shown in Figure 2-left) of the obtained mesoporous polymer (MC773-P1H2) and carbon (MC973-P1H2) both possess two obvious 
diffraction peaks around $1.0^{\circ}$ and $1.5^{\circ}$, which can be indexed as 100 and 110 diffractions of ordered hexagonal mesoporous structure. Upon high temperature carbonization, the obtained MC973-P1H2 possesses weaker secondary diffraction peak than that of MC773-P1H2, implying a reduction of ordering degree of the mesoporous structure. Their ordered mesostructures are further confirmed by the TEM images shown in Figure 3, viewed along [100] and [110] directions. After removal of template at $773 \mathrm{~K}$, well-defined ordered mesoporous structure can be observed with a pore size of $\sim 3.70 \mathrm{~nm}$ (Figure $3 \mathrm{a}$ and $3 \mathrm{~b}$ ). As the calcination temperature increase, ordered mesostructure could be maintained but the ordering degree of the final product of MC973-P1H2 has a slight decrease as the TEM images (Figure 3c and 3d) shown, which is consistent with the results of the small angle XRD patterns.

The structure of MC773-P1H2 and MC973-P1H2 are further characterized by nitrogen sorption isotherms shown in Figure 2-right. Both of them possess a type IV isotherm with a clear condensation step at relative pressure of $0.4-0.6$, which indicates the existence of uniform mesopores. Notably, the adsorption and desorption isotherms of $\mathrm{MC} 773-\mathrm{P} 1 \mathrm{H} 2$ are not closed at the low relative pressures, which are typical isotherms of polymer [35]. The detailed textual properties are listed in Table 1. The specific surface areas (BET) are $423 \mathrm{~m}^{2} / \mathrm{g}$ for MC773-P1H2, $435 \mathrm{~m}^{2} / \mathrm{g}$ for MC973$\mathrm{P} 1 \mathrm{H} 2$ and the corresponding pore volumes are 0.46 and $0.41 \mathrm{~cm}^{3} / \mathrm{g}$, respectively. The Barrett-Joyner-Halenda (BJH) pore size distribution curves, depicted as the inset of Figure 2-right, show that the obtained mesoporous carbons have a narrowly pore size distribution with the average size of $\sim 3.61 \mathrm{~nm}$, consistent with the TEM measurements.

It is anticipated that the $\mathrm{FeOx}$ catalyst would be simultaneously removed by acid dissolution after assembly of precursors and template by phase separation. To examine whether the iron oxide was effectively removed, a Thermogravimetric and Differential 
Scanning Calorimetry analysis (TG-DSC) was done under air flow as shown in Figure 4. TG curve shows that the mass loss mainly occurs at the temperature of $733 \sim 943 \mathrm{~K}$, at which a wide exothermic peak emerged on the DSC curve, indicative of a good thermal stability of the obtained mesoporous carbon. The total loss is near $100 \mathrm{wt} \%$ above the temperature of $973 \mathrm{~K}$, implying that the iron oxide catalyst used in the original catalytic system could be effectively removed.

The above characterizations demonstrate the feasibility of our developed method towards the fabrication of OMC under acidic conditions with phenol as starting material. Obviously, the key to yielding an OMC of our method is the catalytic hydroxylation step. With regard to catalytic hydroxylation, as the Table 2 shown, the selectivity of all products varies with the original ratio of hydrogen peroxide to phenol and this may influence the structure of the final obtained carbon materials. To investigate the effect of the original ratio of $\mathrm{H}_{2} \mathrm{O}_{2}$ /phenol on the final structure, assembly process with different ratio of $\mathrm{H}_{2} \mathrm{O}_{2} /$ phenol in the catalytic step were also operated.

The small angle XRD pattern and nitrogen sorption isotherm of the obtained carbon with the $\mathrm{H} 2 \mathrm{O} 2 /$ phenol molar ratio of 1:1 are shown in Figure $\mathrm{S} 2$. The small angle XRD pattern (Figure S2-left) of MC973-P1H1 shows a very weak and inconspicuous diffraction peak around $1.0^{\circ}$, suggesting the existence of disordered mesoporous structure. The nitrogen sorption isotherm of MC973-P1H1 (Figure S2-right) is a type IV isotherm with a clear condensation step at relative pressures of 0.4-0.6, standing for the existence of uniform mesopore (centered at $3.7 \mathrm{~nm}$ ), which was further confirmed by its pore size distribution curve (Figure S2-right-inset). As the Table 2 shown, the specific surface area of MC973-P1H1 is $407 \mathrm{~m}^{2} / \mathrm{g}$ and the surface area arose from mesoporosity is $177 \mathrm{~m}^{2} / \mathrm{g}$, illustrating the disordered mesoporous structure could be constructed with the $\mathrm{H}_{2} \mathrm{O}_{2}$ /phenol ratio of $1: 1$ by the designed route. The small angle 
XRD pattern of the obtained MC973-P1H3 with $\mathrm{H}_{2} \mathrm{O}_{2} /$ phenol molar ratio of 3:1 (as shown in Figure S3) has a single wide diffraction peak around $1.0^{\circ}$, indicating a mesoporous structure with a poor ordering degree could be obtained. The corresponding nitrogen sorption isotherms (Figure S3-right) show a type IV isotherm with a condensation step at relative pressure of $0.4-0.6$, indicative of the existence of uniform mesopore. The specific surface area for MC973-P1H3 is $410 \mathrm{~m}^{2} / \mathrm{g}$ and the corresponding pore volume is $0.49 \mathrm{~cm}^{3} / \mathrm{g}$. The poor ordering degree of MC973-P1H1 and MC973-P1H3 can be explained by the corresponding products distribution of the initial catalytic hydroxylation as shown in Table 2. The low yield of DBs (41.5\%) at $\mathrm{H}_{2} \mathrm{O}_{2} /$ phenol molar ratio of 1:1 may lead to a low cross-linking degree of the precursor, resulting in the poor ordering degree of MC973-P1H1. In case of $\mathrm{H}_{2} \mathrm{O}_{2} /$ phenol molar ratio up to 3: 1, though the conversion of phenol and the yield of DBs could be enhanced, meanwhile, the yield of benzoquinone increase remarkably, which is unfavorable to form ordered mesostructure. As a result, the obtained MC973-P1H3 possesses a less ordered mesoporous strucutre. So, increasing the amount of $\mathrm{H}_{2} \mathrm{O}_{2}$ in the original catalytic system does not always benefits the assembly process since more byproducts would be generated and the optimal ratio of $\mathrm{H}_{2} \mathrm{O}_{2} /$ phenol is $2: 1$.

\subsection{Electrochemical supercapacitive performances}

The mesoporous carbon with open-accessed and regular arranged mesopores has many potential applications such as catalysis, sorption, electrochemical supercapacitors [36-39] and etc. Here, the electrochemical capacitive performances of MC973-P1H2, ordered mesoporous carbon (OMC973-EISA, BET surface area of $522 \mathrm{~m}^{2} / \mathrm{g}$ ) fabricated by evaporation induced self-assembly method [24] and commercial activated carbon (AC, BET surface area of $891 \mathrm{~m}^{2} / \mathrm{g}$ ) are evaluated. As shown in Figure 5a, the cyclic voltammogram $(\mathrm{CV})$ of MC973-P1H2 exhibits a rectangular-shaped pattern with a 
redox peaks around $0.4 \mathrm{~V}$, indicating that the capacitances of MC973-P1H2 are contributed by the electrochemical double layered capacitive (EDLC) and pseudocapacitive behaviors. Both the CV plots of OMC973-EISA and AC exhibit a rectangular-shaped pattern without any redox signs, showing that the capacitive performance of the two samples are mainly contributed by EDLC. Figure 5b shows that the specific capacitance of MC973-P1H2 far exceeds those of OMC973-EISA and AC at all current densities from 0.5 to $20 \mathrm{~A} / \mathrm{g}$. Notably, the specific capacitance of MC973$\mathrm{P} 1 \mathrm{H} 2$ obtained at $0.5 \mathrm{~A} / \mathrm{g}$ is $231 \mathrm{~F} / \mathrm{g}$, a value higher than OMC973-EISA (151 F/g), AC (119 F/g). Even at high current density of $20 \mathrm{~A} / \mathrm{g}$, a specific capacitance of $175 \mathrm{~F} / \mathrm{g}$ can be maintained, showing an excellent rate capability. Since the electrochemical capacitive behavior occurs on the surface, hence, the surface composition is a key factor to the capacitance. The composition of MC973-P1H2, OMC973-EISA and AC were confirmed by elemental analysis and shown in Table 3. Obviously, compared to OMC973-EISA(O: 10.9 wt. \%), the higher capacitance of MC973-P1H2 (O: 13.1 wt. \%) may be attributed to its higher surface oxygen content, which might be derived from the phenol hydroxylation step. The higher oxygen content not only could enhance the surface wettability [40] but also could introduce pseudocapacitance, leading to a larger capacitance.

The surface chemistry of the three carbons were also investigated by FTIR spectroscopy (Figure 6) and X-ray photoelectron spectra shown in Figure S4. The peak around $1649 \mathrm{~cm}^{-1}$ corresponds to $\mathrm{C}=\mathrm{O}$ stretching vibrations related to carbonylcontaining groups, which are active in electrolyte [41] by Faradic reaction $(-\mathrm{C}=\mathrm{O}+\mathrm{e}-$ $\leftrightarrow$ C-O-) and thus provides pseudocapacitive contributions. Clearly, MC973-P1H2 shows a sharper and larger absorption peak of $\mathrm{C}=\mathrm{O}$ than OMC973-EISA and AC, suggesting the resulted MC973-P1H2 possesses more pseudocapacitive functionalities. 
O1s core level peak of XPS (Figure. S4a) for MC973-P1H2 shows two fitting peaks of $531.2 \mathrm{eV}$ and $532.6 \mathrm{eV}$, assigned to $-\mathrm{C}=\mathrm{O}$ and $-\mathrm{C}-\mathrm{O}$ related groups, respectively [42]. For OMC973-EISA and AC, the O1s core level peaks of XPS (Figure. S4b and S4c) both show three fitting peaks of $531.2 \mathrm{eV}, 532.6 \mathrm{eV}$ and $533.4 \mathrm{eV}$ assigned to $-\mathrm{C}=\mathrm{O}$, $\mathrm{C}-\mathrm{O}$ and $-\mathrm{OH}$ related groups, respectively. As Figure S4d shown, the carbonyl oxygen content for the three carbons calculated from XPS are 6.28 at.\% (MC973-P1H2), 2.62 at.\% (OMC973-EISA) and 1.93 at.\% (AC), which further confirms that the resulted MC973-P1H2 possesses more pseudocapacitive functionalities. In the other words, the developed route favors the introduction of oxygenic functionalities on mesoporous carbon, which brings a higher capacitance than normal mesoporous carbon. Figure 5c shows the galvanostatic charge/discharge curves of MC973-P1H2 under various current densities and all the curves exhibits a regular triangular shaped pattern, revealing good columbic efficiency. In order to evaluate the electrochemical cyclic stability of the MC973-P1H2, galvanostatic charge/discharge investigations were performed at a high current density of $10.0 \mathrm{~A} / \mathrm{g}$. As shown in Figure 5d, after 5000 cycles, the MC973P1H2 remains a high specific capacitance of $194 \mathrm{~F} / \mathrm{g}$ and the retention is about $98 \%$, indicative of an excellent electrochemical cycleability. The superior ECs performance of MC973-P1H2 to normal OMC and AC mainly attributed to the abundant oxygenic functionalities resulted from catalytic hydroxylation, thus providing pseudocapacitance and leading to higher capacitance.

\section{Conclusions}

A synthesis methodology combined phenol catalytic hydroxylation and coassembly process has been successfully developed to aqueous fabrication of ordered mesoporous carbon under highly acidic conditions with phenol as starting material. 
Phenol was first partially converted into highly reactive phenols (catechol and hydroquinone) and then the resulted mixture (catechol, hydroquinone and phenol) interacted with formaldehyde and template through enhanced hydrogen bonding and electrostatic interaction under highly acidic conditions to form a stable mesophase by phase separation and ordered mesoporous carbon was successfully obtained after carbonization. Moreover, the obtained oxygen-rich ordered mesoporous carbon demonstrates superior electrochemical capacitive performances $(231 \mathrm{~F} / \mathrm{g})$ to normal ordered mesoporous carbon (151 F/g) and commercial activated carbon (119 F/g). The current synthesized ordered mesoporous carbon may find utility in many other applications e.g. catalysis, adsorption, etc. The developed strategy not only affords a novel approach for the synthesis of OMC from cheap phenol under acidic condition but also provides a way for the direct functionalization of OMC, e.g. in situ introducing pseudocapacitive oxygen on ordered mesoporous carbon.

\section{Acknowledgment:}

This work was supported by the Ministry of Science and Technology of China (2009CB623504), the National Science Foundation of China (20773062, 20773063, 21173119, and 21273109), the Fundamental Research Funds for the Central Universities and the Postdoctoral Foundation of Jiangsu (0205003455). The authors also thank the support from the Shanghai Research Institute of Petrochemical Technology, Sinopec.

\section{References}

[1] R. Ryoo, SH. Joo, S. Jun, J. Phys. Chem. B. 103 (1999) 7743-7746.

[2] A. H. Lu, B. Spliethoff, F. Schueth, Chem. Mater. 20 (2008) 5314-5319. 
[3] D. Liu, J. H. Lei, L. P. Guo, D. Qu, Y. Li, B. L. Su, Carbon. 50 (2012) 476-487.

[4] M. Xie, Y. Xia, J. Liang, L. Chen, X. Guo, Micropor. Mesopor. Mater. 197 (2014) $237-243$

[5] Q. Shi, R. Zhang, Y. Lu, Y. Deng, A. A. Elzatahrya, D. Y. Zhao. Carbon. 84 (2015) $335-46$.

[6] Z. Dong, C. Dong, Y. Liu, X. Le, Z. Jin, J. Ma, Chem. Eng. J. 270 (2015) 215-222.

[7] M. Zhang, A. Sun, Y. Meng, L. Wang, H. Jiang, G. Li, Micropor. Mesopor. Mater. 204 (2015) 210-217.

[8] F. Sun, J. Liu, H. Chen, Z. Zhang, W. Qiao, D. Long, et al. ACS Catal. 3 (2013) 862-870.

[9] Z. Sun, B. Sun, M. Qiao, J. Wei, Q. Yue, C. Wang, et al. J. Am. Chem. Soc.134 (2012) 17653-17660.

[10]M. Barczak, K. Michalak-Zwierz, K. Gdula, K. Tyszczuk-Rotko, R. Dobrowolski, A. Nbrowski, Micropor. Mesopor. Mater. 211 (2015) 162-173.

[11]B. Yuan, X. Wu, Y. Chen, J. Huang, H. Luo, S. Deng, Environ. Sci. Technol. 47 (2013) 5474-5480.

[12]J. Balach, T. Jaumann, M. Klose, S. Oswald, J. Eckert, L. Giebeler, J. Phys. Chem. C. 119 (2015) 4580-4587.

[13] Y. Liu, J. Zhang,Micropor. Mesopor. Mater. 206 (2015) 81-85.

[14]Y. Lin, X. Wang, G. Qian, J. J. Watkins. Chem. Mater. 26 (2014) 2128-2137.

[15]T. Y. Ma, L. Liu, Z. Y Yuan, Chem. Soc. Rev. 42 (2013) 3977-4003.

[16] Q. Wang, W. Zhang, Y. Mu, L. Zhong, Y. Meng, Y. Sun, Micropor. Mesopor. Mater. (2014) 197 109-115.

[17] C. D. Liang, S. Dai, J. Am. Chem. Soc.128(2006):5316-5317.

[18]C. Liu, L. Li, H. Song, X. Chen. Chem. Commun. (2007) 757-759. 
[19]D. Liu, L. J. Xia, D. Qu, J. H Lei, Y. Li, B. L Su. J. Mater. Chem. A. 1 (2013) 15447-15458.

[20]L. Liu, Q. F. Deng, T. Y Ma, X. Z Lin, X. X Hou, Y. P Liu, et al. J. Mater. Chem. 21 (2011) 16001-16009.

[21] R. T. Mayes, C. Tsouris, JO. Kiggans, S. M. Mahurin, D. W. DePaoli, S. Dai, J. Mater. Chem. 20 (2010) 8674-8678.

[22]X. Wang, C. Liang, S. Dai, Langmuir. 24 (2008) 7500-7505.

[23]Y. Liang, R. Fu, D. Wu, ACS Nano.7 (2013) 1748-1754.

[24]M. Xie, H. Dong, D. Zhang, X. Guo, W. Ding, Carbon. 49 (2011) 2459-2464.

[25]F. Zhang, Y. Meng, D. Gu, Y. Yan, Z. Chen, B. Tu, et al. Chem. Mater. 18 (2006) $5279-5288$.

[26]F. Q. Zhang, Y. Meng, D. Gu, Y. Yan, C. Z. Yu, B. Tu, et al. J. Am. Chem. Soc. 127 (2005) 13508-13509.

[27]CCM. Ma, H. D. Wu, P. P Chu, H. T. Tseng, Macromol. 30 (1997) 5443-5449.

[28]F. Y. Wang, CCM. Ma, H. D. Wu, J. Appl. Poly. Sci. 74 (1999) 2283-2289.

[29]Y. Huang, J. Yang, H. Cai, Y. Zhai, D. Feng, Deng Y, et al. J. Mater. Chem. 19 (2009) 6536-6541.

[30]H. Kosonen, S. Valkama, A. Nykanen, M. Toivanen, G. Ten Brinke, J. Ruokolainen, et al. Adv. Mater. 18 (2006) 201-205.

[31]X. Zhao, A. Wang, J. Yan, G. Sun, L. Sun, T. Zhang, Chem. Mater. 22 (2010) 5463 5473.

[32]P. Gao, A. Wang, X. Wang, T. Zhang. Chem. Mater. 20 (2008) 1881-1888.

[33] J. Song, Y. Zhang, Y. Liu, Rsc Adv. 5 (2015) 20734-20740.

[34]C. P. Huang, Y. H. Huang. Appl. Catal. A. 346 (2008) 140-148.

[35]Y. Meng, D. Gu, F. Zhang, Y. Shi, L. Cheng, D. Feng, et al. Chem. Mater. 18 (2006) 
4447-4464.

[36]T. Cai, M. Zhou, D. Ren, G. Han, S. Guan, J Power Sources. 231 (2013) 197-202.

[37]G. Sun, J. Wang, X. Liu, D. Long, W. Qiao, L. Ling. J. Phys. Chem. C. 114 (2010) 18745-18751.

[38] S.Tanaka, A. Doi, T. Matsui, Y. Miyake. J Power Sources. 228 (2013) 24-31.

[39]D. D. Zhou, Y. J. Du, Y. F Song, Y. G Wang, C. X. Wang, Y. Y. Xia, J. Mater. Chem.

A.1 (2013) 1192-200.

[40]M. Seredych, D. Hulicova-Jurcakova, G. Q. Lu, T. J. Bandosz. Carbon.46 (2008) 1475-1488.

[41] J. W. Lang, X. B. Yan, W. W. Liu, R. T. Wang, Q. J. Xue. J. Power Sources. 204 (2012) 220-229.

[42] C.-M. Chen, Q. Zhang, X.-C. Zhao, B. Zhang, Q.-Q. Kong, M.-G. Yang, Q.-H. Yang, M.-Z. Wang, Y.-G. Yang, R. Schloegl, D.S. Su. J. Mater. Chem. 22 (2012) 1407614084. 


\section{Tables}

Table 1. Textural properties of MCT-PxHy.

\begin{tabular}{|c|c|c|c|c|c|}
\hline Sample & $\begin{array}{c}\mathrm{S}_{\mathrm{BET}} \\
\left(\mathrm{m}^{2} \mathrm{~g}^{-1}\right)\end{array}$ & $\begin{array}{c}\mathrm{V}_{\text {total }} \\
\left(\mathrm{cm}^{3} \mathrm{~g}^{-1}\right)\end{array}$ & $\begin{array}{l}\text { Pore size } \\
\text { (nm) }\end{array}$ & $\begin{array}{l}\mathrm{S}_{\text {Micro }} \\
\left(\mathrm{m}^{2} \mathrm{~g}^{-1}\right)\end{array}$ & $\begin{array}{l}\mathrm{S}^{\mathrm{a}} \text { Exter } \\
\left(\mathrm{m}^{2} \mathrm{~g}^{-1}\right)\end{array}$ \\
\hline MC973-P1H0 & 421 & 0.25 & - & 381 & 40 \\
\hline MC973-P1H1 & 407 & 0.39 & 3.7 & 230 & 177 \\
\hline MC773-P1H2 & 423 & 0.46 & 3.7 & 238 & 185 \\
\hline MC973-P1H2 & 435 & 0.41 & 3.8 & 298 & 137 \\
\hline MC973-P1H3 & 410 & 0.49 & 3.9 & 260 & 149 \\
\hline
\end{tabular}


Table 2. Products distribution after catalytic phenol hydroxylation for $12 \mathrm{~h}$ with different molar ratio of phenol to hydrogen peroxide.

\begin{tabular}{ccccccc}
\hline Sample & Yield (\%) & Conversion (\%) & \multicolumn{3}{c}{ Selectivity (\%) } \\
& $\mathrm{DB}^{\mathrm{a}}$ & $\mathrm{PH}^{\mathrm{b}}$ & $\mathrm{CT}^{\mathrm{c}}$ & $\mathrm{HQ}^{\mathrm{d}}$ & $\mathrm{BQ}^{\mathrm{e}}$ \\
MC973-P1H1 & 41.5 & 42.1 & 46.9 & 51.6 & 1.06 \\
MC973-P1H2 & 52.3 & 54.2 & 36.1 & 60.5 & 2.45 \\
& & & & & & \\
MC973-P1H3 & 54.6 & 60.5 & 21.1 & 69.2 & 7.90
\end{tabular}

a: Dihydroxybenzene (Catechol and Hydroquinone); b: Phenol; c: Catechol; d: Hydroquinone; e: Benzoquinone.

Table 3. Elemental analysis results of MC973-P1H2, OMC973-EISA and AC.

$\begin{array}{cccccc}\text { Sample } & \mathrm{C}^{\mathrm{a}} & \mathrm{H}^{\mathrm{a}} & \mathrm{N}^{\mathrm{a}} & \mathrm{S}^{\mathrm{a}} & \mathrm{O}^{\mathrm{b}} \\ \text { MC973-P1H2 } & 86.5 & 0.41 & 0 & 0 & 13.1 \\ \text { OMC973-EISA } & 88.4 & 0.63 & 0 & 0 & 10.9 \\ \text { AC } & 90.2 & 0.23 & 0.85 & 1.1 & 7.6\end{array}$

a: The contents of $\mathrm{C}, \mathrm{H}$, and $\mathrm{N}$ were measured by elemental analysis. $\mathrm{b}$ : the oxygen contents were calculated by considering that $\mathrm{O}$ is the only remaining element in the samples. 


\section{Figure captions:}

Figure 1. Schematic diagram of phenol catalytic hydroxylation and assembly with template to ordered mesoporous carbon.

Figure 2. Small angle XRD patterns (left), nitrogen sorption isotherm and pore size distribution curves (right) of MCT-PxHy.

Figure 3. TEM images of MC773-P1H2 a, b); and MC973-P1H2, c, d) viewed along [100] (a, c) and [110] (b, d) directions.

Figure 4. Differential scanning calorimeter (DSC) curves and thermo-gravimetric analysis of MC973-P1H2 under air atmosphere.

Figure 5. Electrochemical capacitive performance of the obtained MC973-P1H2, OMC973-EISA and Activated Carbon (AC) in $1.0 \mathrm{M} \mathrm{H}_{2} \mathrm{SO}_{4}$, a) Cylic voltammogram of MC973-P1H2, OMC973-EISA and AC , b) specific capacitance of MC973-P1H2, OMC973-EISA and AC under various current densities, c) galvanostatic charge/discharge curves of MC973-P1H2 under various current densities, d) Cycle stability test of MC973-P1H2 under current density of $10 \mathrm{~A} / \mathrm{g}$.

Figure 6. FTIR spectra of MC973-P1H2, OMC973 and AC. 


\section{Figures}

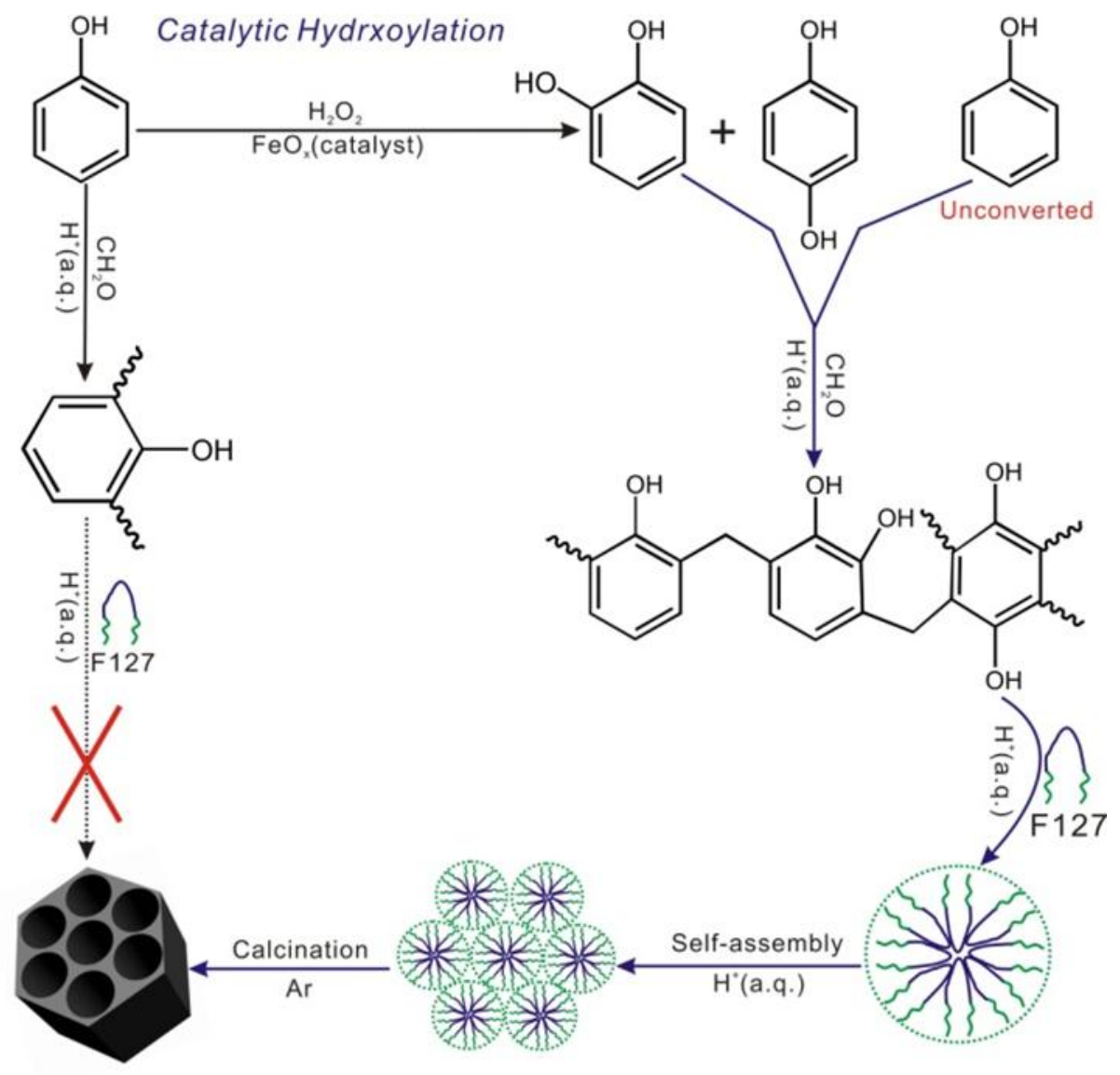

Figure 1. Schematic diagram of phenol catalytic hydroxylation and assembly with template to ordered mesoporous carbon. 

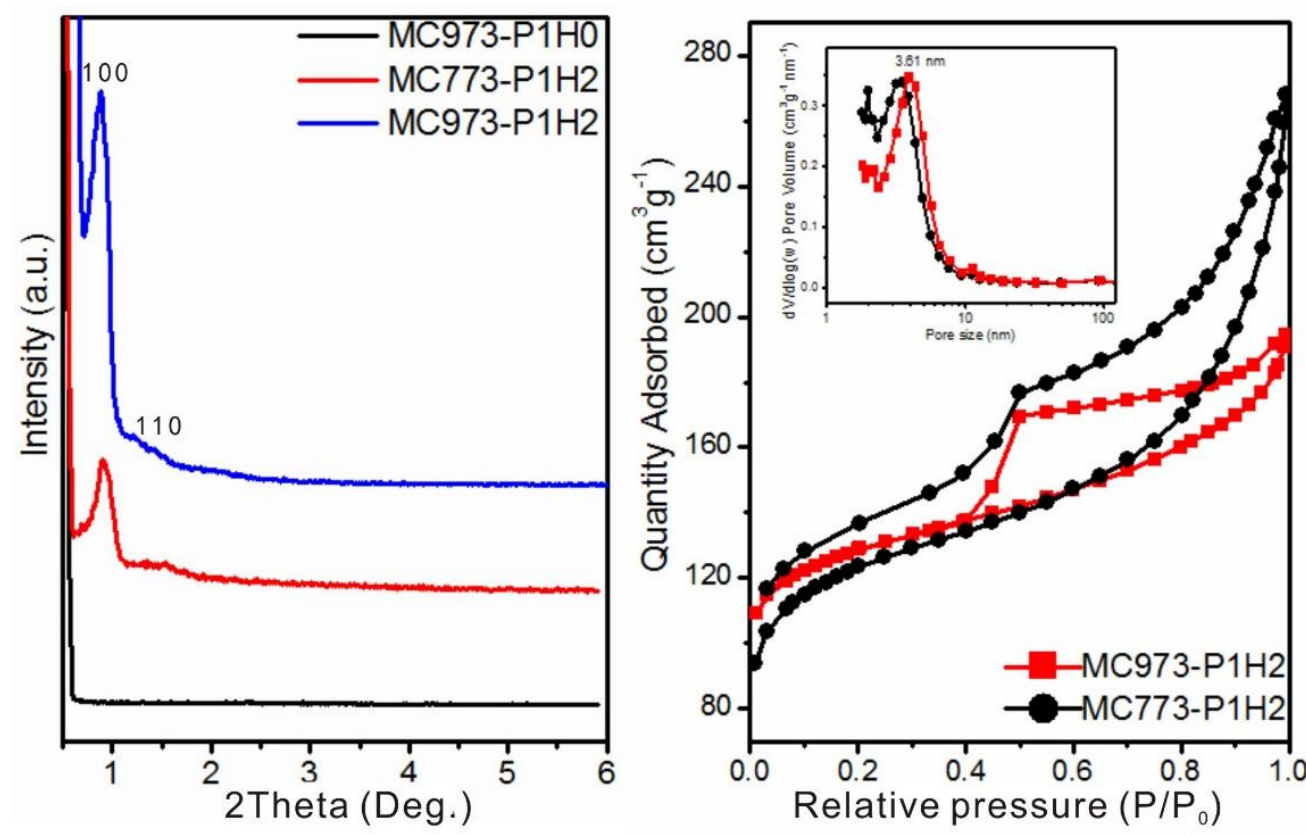

Figure 2. Small angle XRD patterns (left), nitrogen sorption isotherm and pore size distribution curves (right) of MCT-PxHy.

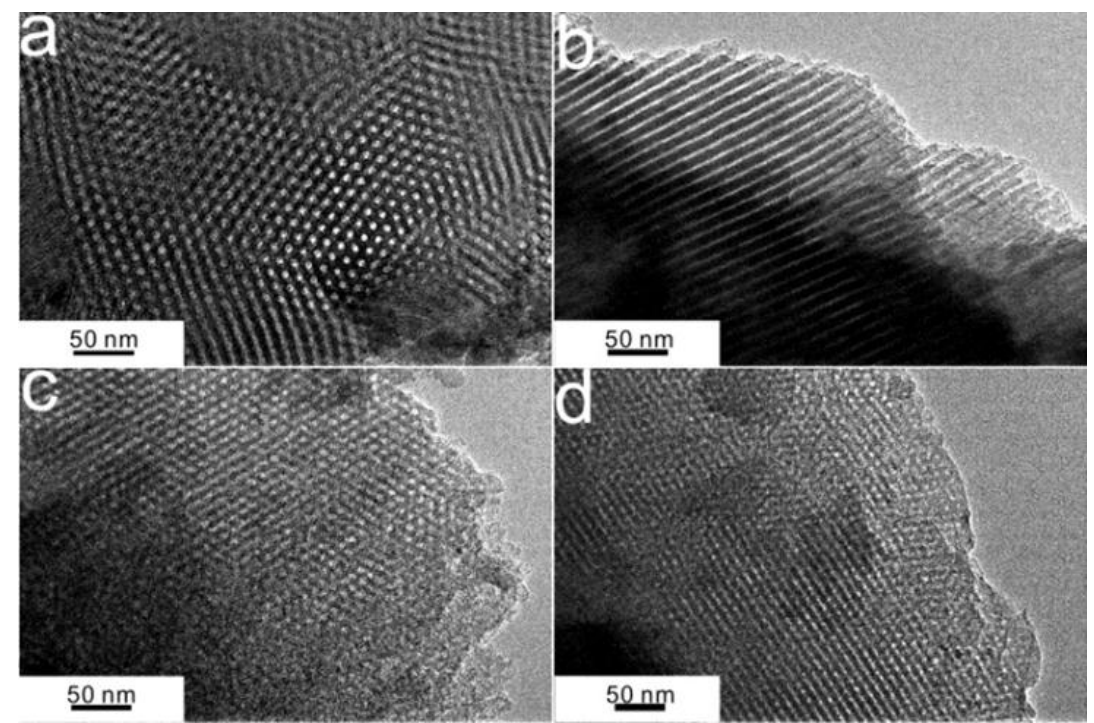

Figure 3. TEM images of MC773-P1H2 a, b); and MC973-P1H2, c, d) viewed along [100] (a, c) and [110] (b, d) directions. 


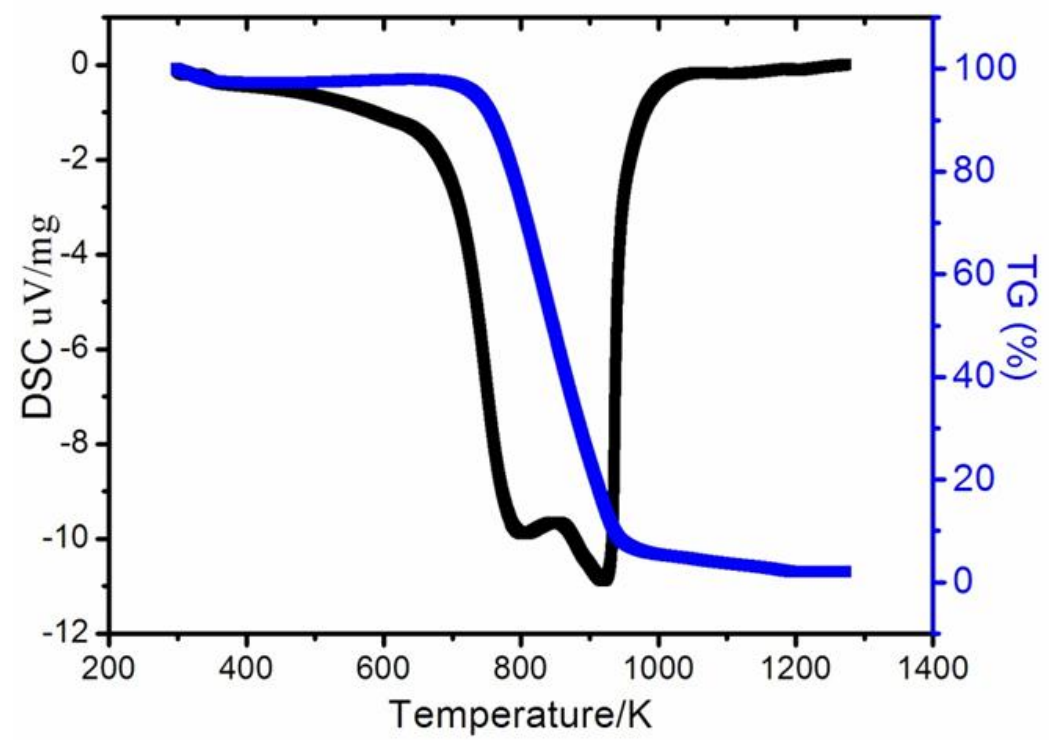

Figure 4. Differential scanning calorimeter (DSC) curves and thermo-gravimetric analysis of MC973-P1H2 under air atmosphere. 

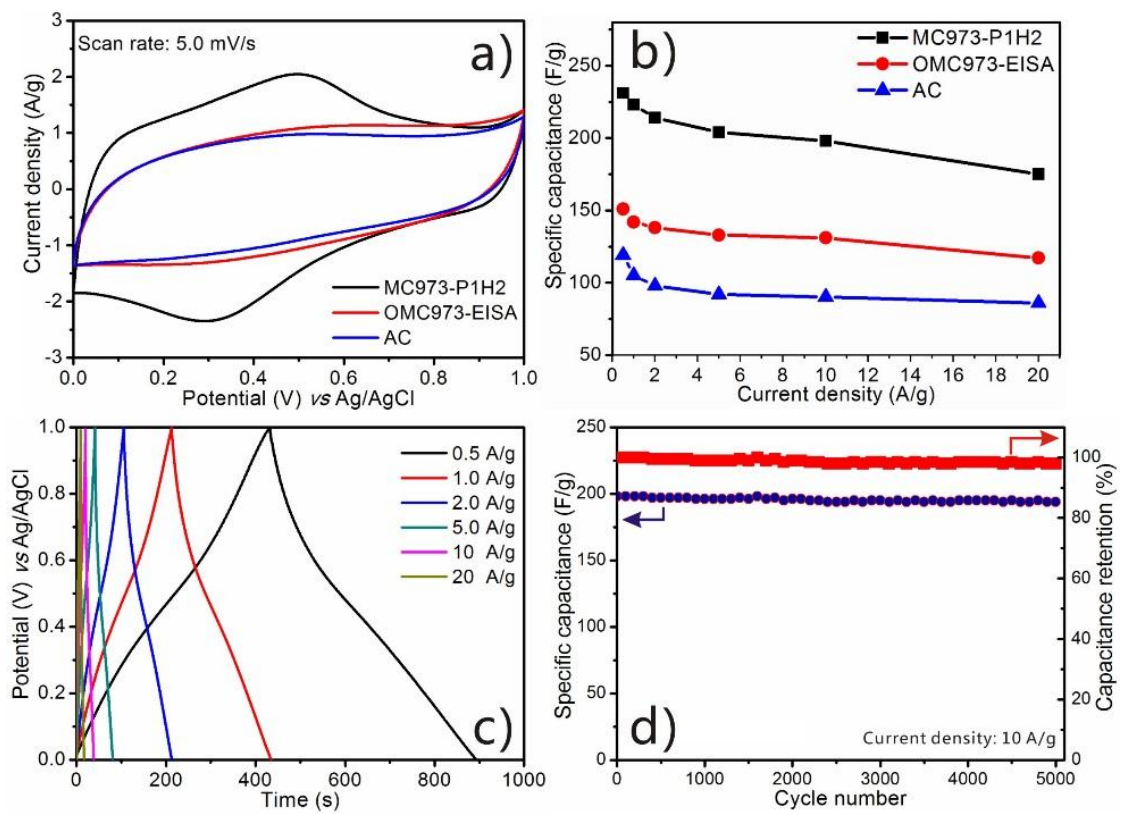

Figure 5. Electrochemical capacitive performance of the obtained MC973-P1H2, OMC973-EISA and Activated Carbon (AC) in 1.0 M H2SO4, a) Cylic voltammogram of MC973-P1H2, OMC973-EISA and AC , b) specific capacitance of MC973-P1H2, OMC973-EISA and AC under various current densities, c) galvanostatic charge/discharge curves of MC973-P1H2 under various current densities, d) Cycle stability test of MC973-P1H2 under current density of $10 \mathrm{~A} / \mathrm{g}$. 


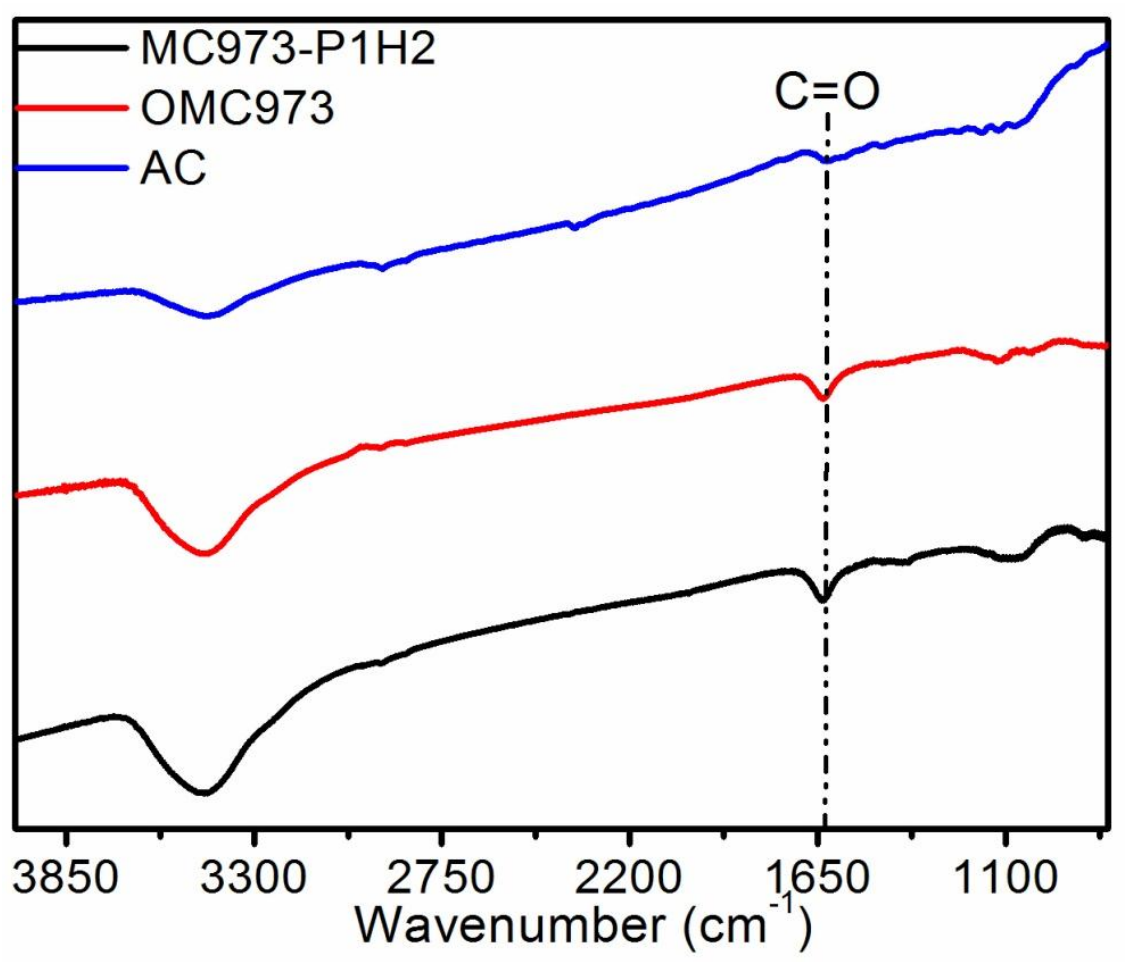

Figure 6. FTIR spectra of MC973-P1H2, OMC973 and AC. 
Graphical abstract:

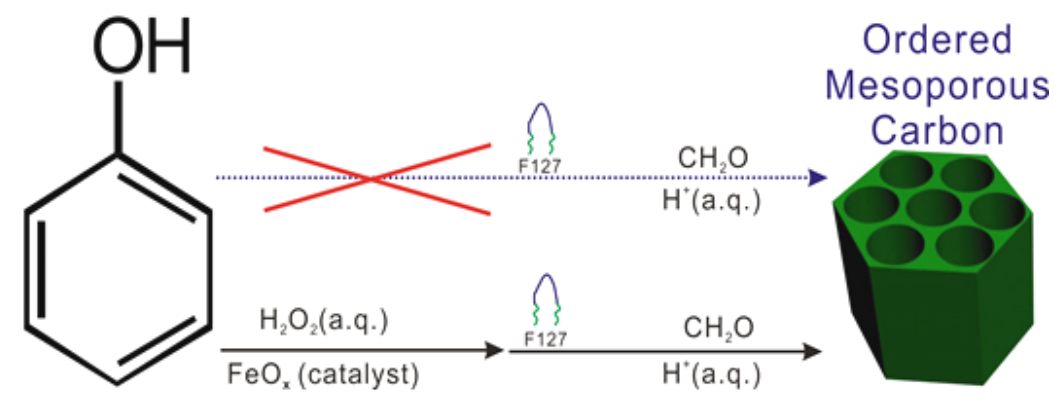

